\title{
EFEITO DE DOSES DE SILÍCIO SOBRE A PRODUTIVIDADE E CARACTERÍSTICAS AGRONÔMICAS DA SOJA [Glycine max (L.) Merrill]
}

\author{
Effects of doses of silicon in the yield and agronomic characteristics \\ of soybean [Glycine max (L.) Merrill]
}

\author{
Péricles Pereira Júnior ${ }^{1}$, Pedro Milanez Rezende ${ }^{2}$, Stephan Carvalho Malfitano ${ }^{3}$, \\ Rafaela Karin Lima ${ }^{4}$, Luíza Vasconselos Tavares Corrêa ${ }^{2}$, Evrerson Reis Carvalho ${ }^{2}$
}

\begin{abstract}
RESUMO
A soja [Glycine max (L.) Merrill] é o principal produto agrícola da exportação brasileira, portanto, torna-se importante a realização de estudos que contribuam para a minimização dos efeitos que possam causar a diminuição do rendimento e a depreciação da qualidade dessa oleaginosa, como a ocorrência de pragas e doenças, problemas com a fertilidade do solo e adversidades climáticas, entre outros. Sabe-se que as plantas diferem na sua capacidade de absorver silício ( $\mathrm{Si}$ ), sendo assim diferentemente classificadas. A influência do Si no desenvolvimento vegetativo em plantas de soja ainda é pouco conhecida, existindo poucos estudos descritos na literatura. Diante disso, objetivou-se, neste trabalho, avaliar a resposta da soja a diferentes doses de Si no plantio, por meio da produtividade e características agronômicas das plantas no campo. O delineamento experimental utilizado foi o de blocos casualizados (DBC), com três repetições, tendo como tratamentos 11 doses de Si $\left(0,50,100,150,200,250,300,350,400,450\right.$ e $\left.500 \mathrm{~kg} \mathrm{ha}^{-1}\right) \mathrm{na}$ semeadura da soja, cultivar BRS MG 68 Vencedora. As doses de Si utilizadas não proporcionaram aumentos significativos na produtividade de grãos, peso de mil sementes, número de sementes por legume, o mesmo não se verificando com o numero de legumes por planta, altura de planta e altura de inserção do primeiro legume que apresentaram efeito significativo, com o aumento das doses nessas características. A aplicação de Si não proporcionou efeitos fitotóxicos visíveis na planta de soja.
\end{abstract}

Termos para indexação: Silicato, adubação, soja.

\section{ABSTRACT}

Soybean [Glycine $\max (\mathrm{L}$.$) Merrill] is the main agricultural product export of Brazil. Therefore, it is important studies that$ contribute to the mitigation of the effects that might cause a decrease in income and depreciation of the quality of this oleaginous plant, as the occurrence of pests and diseases, problems with soil fertility and climatic adversities, among others. It is known that plants differ in their ability to absorb silicon $(\mathrm{Si})$ and are therefore classified differently. The influence of vegetation on $\mathrm{Si}$ in the development of soybean plants is still little known, and there are few studies described in the literature. Facing this, the objective of this work was to evaluate the response of soybean plants to variable dosage of silicon in the plantation, through agronomical characteristics of the plants in the field. The experimental design was a completely randomized block (CBD), with three replicates, eleven treatments with the following doses of silicon $\left(0,50,100,150,200,250,300,350,400,450\right.$ and $\left.500 \mathrm{~kg} \mathrm{ha}^{-1}\right)$ in the soybean planting, cultivating BRS MG 68 Vencedora. Doses of silicon used did not provide significant increases in seed yield, weight of a thousand seeds, and number of seeds per vegetable, but the number of vegetables per plant, plant height and time of insertion of the first vegetable increased significantly with higher doses. Application of silicon did not have phytotoxic effects on the soybean plant.

Index terms: silicon, fertilization, soybean.

(Recebido em 23 de abril de 2008 e aprovado em 3 de julho de 2009)

\section{INTRODUÇÃO}

A soja [Glycine $\max ($ L.) Merril] é o principal produto agrícola da exportação brasileira, sendo que a produção nacional na safra 2005/2006 foi de 55 milhões de toneladas (Companhia Nacional de Abastecimento - CONAB, 2007). Diante disso, torna-se importante a realização de estudos que contribuam para a minimização dos efeitos que possam causar a diminuição do rendimento e a depreciação da qualidade dessa oleaginosa, como a ocorrência de pragas e doenças, problemas com a fertilidade do solo e adversidades climáticas, entre outros. $\mathrm{O}$ fornecimento de insumos adequados, como os nutrientes necessários durante todo o ciclo da cultura, são de importância inquestionável. Assim, o estudo sobre a utilização de $\mathrm{Si}$ (Si), nessa cultura, torna-se necessário por apresentar potenciais benefícios para a planta, sendo reconhecidas sua influência na resistência das plantas ao ataques de insetos, nematóides, bactérias e fungos, na melhoria do

'Universidade Federal de Lavras/UFLA - Departamento de Agricultura/DAG - Cx.P. 3037 - 37200-000 - Lavras, MG - jrpericlespereira@yahoo.com.br 2Universidade Federal de Lavras/UFLA - Departamento de Agricultura/DAG - Lavras, MG

${ }^{3}$ Universidade Federal de Lavras/UFLA - Departamento de Entomologia/DEN - Lavras, MG

${ }^{4}$ Universidade Federal de Lavras/UFLA - Departamento de Química/DQI - Lavras, MG 
estado nutricional, na redução da transpiração e, possivelmente, também em alguns aspectos da eficiência fotossintética.

Tem-se notado alguns benefícios utilizando esse elemento no solo e em solução nutritiva, como a redução dos efeitos tóxicos de ferro, manganês e alumínio, conforme relata o trabalho de Ma \& Takahashi (1991). Alguns autores têm sugerido que o fornecimento de $\mathrm{Si}$ às plantas pode aliviar a toxidez de Mn e Fe não somente pela redução na sua absorção, mas também porque aumenta o nível de tolerância interna ao excesso de Mn nos tecidos (Savant et al., 1999).

Os efeitos benéficos do Si têm sido demonstrados em várias espécies vegetais, especialmente quando essas plantas são submetidas a algum tipo de estresse, seja ele de caráter biótico ou abiótico (Datnoff et al., 2001). Quanto à sua absorção, a soja se classifica em intermediária, a qual apresenta quantidade considerável de $\mathrm{Si}$, quando a concentração do elemento no meio é alta, pois transloca livremente das raízes para a parte aérea (Miyake \& Takahashi, 1985).

Esse elemento pode estimular o crescimento e a produção vegetal por meio da formação de folhas mais eretas, com a consequente diminuição do auto-sombreamento; por meio da redução do acamamento; pela maior rigidez estrutural dos tecidos; pela proteção contra estresses abióticos tais como amenização da toxidez de $\mathrm{Fe}, \mathrm{Mn}, \mathrm{Al}$ e $\mathrm{Na}$; aumento da tolerância ao estresse hídrico e à geada; pela proteção contra estresses bióticos, tais como a redução do ataque de patógenos (doenças) e aumento na proteção contra herbívoros, incluindo os insetos fitófagos (Marschner, 1995).

Outros estudos com Si realizados em soja estão principalmente relacionados com algumas doenças e pragas. Lima (1998) observou que o aumento da resistência da soja ao cancro da haste pode ser induzido, em condições de hidroponia, por meio do incremento do teor de $\mathrm{Si}$. Verificou também que ocorrem diferenças varietais significativas em relação à absorção de Si por plantas de soja. Juliatti et al. (1996) verificaram a redução da infecção por cancro da haste em diferentes cultivares de soja pela aplicação de wollastonita via solo. Pulverizações em plantas de soja com silicato de potássio, mistura de silicato com fungicida protetor (mancozeb) e mistura de silicato com fungicida sistêmico (epoxiconazole + piraclostrobin) reduziram a severidade da ferrugem asiática (Phakopsora pachyrhizi) em $34 \%, 76 \%$ e $83,3 \%$, respectivamente (Nascimento et al., 2005).

Lima (2006) verificou a redução em $24,5 \%$ da severidade da ferrugem da soja em plantas supridas com silicato de potássio, bem como o aumento do conteúdo de clorofila b e carotenóides, do teor de lignina das folhas, observando também a camada de cera epicuticular mais desenvolvida. No mesmo ano, Ferreira (2006) observou comportamento diferente de cultivares de soja com a aplicação de Si em vasos, sendo que na cultivar IAC-19 houve aumento do grau de resistência à mosca-branca (Bemisia tabaci) biótipo B, ocorrendo também a potencialização da produção de nitrogênio não-protéico em plantas de soja associada à mesma cultivar. Anteriormente, Nolla et al. (2004) observaram que a aplicação de silicato aumentou a concentração de Si foliar em soja.

Neste trabalho, objetivou-se avaliar a resposta das doses crescentes de $\mathrm{Si}$, na produtividade e características agronômicas da soja.

\section{MATERIAL E MÉTODOS}

O experimento foi conduzido na Fazenda Milanez, situada no município de Itutinga no Estado de Minas Gerais, Brasil, na latitude de $21^{\circ} 23^{\prime} 29,8^{\prime \prime S}$, longitude de 4439' 13,2" e altitude média de $958 \mathrm{~m}$. O solo utilizado foi classificado com do tipo Cambissolo, de textura argilosa, com as seguintes características químicas, apresentadas (Tabela 1). As determinações químicas foram realizadas conforme recomendação da Empresa Brasileira de Pesquisa Agropecuária - Embrapa (1997) e o Si de acordo com Kilmer (1965), sendo esse elemento considerado baixo.

O clima da região é classificado segundo Köppen como do tipo Cwa, temperado úmido (com verão quente e inverno seco), caracterizado por um total de chuvas de $23,4 \mathrm{~mm}$ no mês mais seco e de $295,8 \mathrm{~mm}$ no mês mais chuvoso. A temperatura média é de $22,1^{\circ} \mathrm{C}$ no mês mais quente e de $15,8^{\circ} \mathrm{C}$ no mês mais frio, sendo a precipitação média anual de 1529,7 mm (Brasil, 1992).

Tabela 1 - Características químicas do solo amostrado da área experimental.

\begin{tabular}{cccccccccccccccccccc}
\hline $\mathrm{H}_{2} \mathrm{O}$ & $\mathrm{mg} \mathrm{dm}^{-3}$ & \multicolumn{4}{c}{$\mathrm{cmols} \mathrm{dm}^{-3}$} & \multicolumn{4}{c}{$\mathrm{dag} \mathrm{kg}^{-3}$} & $\mathrm{mg} \mathrm{L}^{-1}$ & \multicolumn{4}{c}{$\mathrm{mg} \mathrm{dm}^{-3}$} & & & $\%$ \\
\hline $\mathrm{pH}$ & $\mathrm{P}$ & $\mathrm{K}$ & $\mathrm{Ca}$ & $\mathrm{Mg}$ & $\mathrm{Al}$ & $\mathrm{H}+\mathrm{Al}$ & $\mathrm{SB}$ & $\mathrm{t}$ & $\mathrm{T}$ & $\mathrm{MO}$ & $\mathrm{P} \mathrm{rem}$ & $\mathrm{Zn}$ & $\mathrm{Fe}$ & $\mathrm{Mn}$ & $\mathrm{Cu}$ & $\mathrm{B}$ & $\mathrm{S}$ & $\mathrm{Si}$ & $\mathrm{V}$ \\
\hline 5,5 & 2,5 & 145 & 2,6 & 0,7 & 0 & 3,2 & 3,7 & 3,7 & 6,9 & 3,4 & 7,5 & 1,0 & 45,4 & 9,7 & 4,4 & 0,2 & 42,5 & 0,3 & 53,4 \\
\hline
\end{tabular}

Análise de solo realizada no Instituto de Química “John Wheelock” do Departamento de Ciência do Solo da Universidade Federal de Lavras, Minas Gerais. 
O delineamento experimental utilizado foi o de blocos casualizados (DBC), com três repetições, tendo como tratamentos onze doses de $\mathrm{Si}(0,50,100,150,200$,

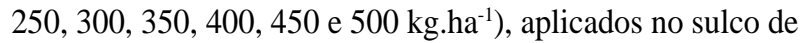
plantio por ocasião da semeadura da soja, totalizando 11 tratamentos. Essas dosagens foram determinadas, levando em conta o nível baixo do solo e o aspecto econômico.

O presente trabalho foi conduzido em solo corrigido considerado de média a alta fertilidade, com dois anos de cultivo. O preparo de solo foi realizado por meio de uma aração e duas gradagens utilizando o sistema convencional. A adubação de semeadura foi realizada de acordo com a análise de solo e as interpretações de acordo com Ribeiro et al. (1999), utilizou-se $120 \mathrm{~kg}$ de $\mathrm{P}_{2} \mathrm{O}_{5}$ na forma de super fosfato simples e $60 \mathrm{~kg}$ de $\mathrm{K}_{2} \mathrm{O} \mathrm{ha}{ }^{-1}$ na forma de cloreto de potássio.

A cultivar utilizada foi a BRS MG 68 (Vencedora) decorrente de seu alto potencial produtivo no cenário da sojicultura nacional e do Estado de Minas Gerais. Pertence ao grupo de maturação médio (130 a 140 dias), exigente em fertilidade de solo, de crescimento determinado, hilo de cor preta, pubescência marrom e resistente ao acamamento. Em trabalhos anteriores, essa cultivar foi destaque com produtividade de $4.395 \mathrm{~kg} \mathrm{ha}^{-1}$ (Rezende \& Carvalho, 2007).

As parcelas no campo foram constituídas de quatro fileiras de 5,0 $\mathrm{m}$ de comprimento espaçadas de $0,5 \mathrm{~m}$ totalizando $10,0 \mathrm{~m}^{2}$. Como área útil, foram utilizadas as duas fileiras centrais, retirando-se ainda $0,50 \mathrm{~m}$ de cada extremidade a título de bordadura correspondendo a $4,0 \mathrm{~m}^{2}$.

As sementes de soja foram inoculadas antes da semeadura com Bradyrhizobium japonicum, utilizandose o inoculante Nitral@ na concentração mínima de 1.200.000 de bactérias por sementes. A semeadura foi realizada no dia 05/12/2005, a uma profundidade de 2 a 3 cm no início do período chuvoso do ano agrícola 2005/ 2006. Verificou-se uma emergência uniforme e vigorosa cerca de cinco dias após a semeadura. Aos quinze dias após a emergência, foi realizado um desbaste uniformizando o estande inicial em 15 plantas por metro linear de acordo com Rezende et al. (1981), buscando-se uma população ideal de 300.000 plantas por hectare. Em fevereiro de 2006, foi utilizado o produto comercial FICAM $®$ (Bendiocarb) para controle de pragas, tendo-se verificado alto nível de vaquinhas (Diabrotica speciosa). Fez-se necessária à utilização de fungicida para controle da Ferrugem Asiática e complexo de doenças do final de ciclo, utilizando-se apenas uma dose do fungicida Óperaâ de $0.5 \mathrm{~L} \mathrm{ha}^{-1}$. O controle das plantas invasoras foi realizado por meio de capina manual a fim de evitar mato competição.
A colheita foi realizada manualmente, quando $95 \%$ dos legumes atingiram a coloração característica de legumes maduros, no estádio R8. Após a colheita, as sementes foram trilhadas e acondicionadas em sacos de papel, realizando-se, posteriormente, a determinação da produtividade, e a pesagem de mil sementes, conforme as regras de análises de sementes no RAS (Brasil, 1992).

Na ocasião da colheita, foram avaliadas nas áreas úteis de todas as parcelas as seguintes características: produtividade de grãos (corrigida a umidade dos grãos para $13 \%$ ) e, posteriormente, transformados para $\mathrm{kg} \mathrm{ha}^{-1}$; altura da planta e inserção do $1^{\circ}$ legume (expressos em $\mathrm{cm}$ ), por meio da avaliação realizada em dez plantas. Determinou-se o número de legumes por planta, número de sementes por legume, sendo essas características avaliadas mediante a contagem de cinco plantas tomadas ao acaso, nas duas linhas centrais úteis de cada parcela. A fonte de Si utilizado foi o Extragan-Si-plus GR fornecido pela Extrativa Fertilizantes S/A com $25 \%$ de $\mathrm{SiO}_{2}$, aplicado no sulco da semeadura misturado aos adubos superfosfato simples e cloreto de potássio.

\section{RESULTADOS E DISCUSSÃO}

Não ocorreu diferença estatística para as características na produtividade (PRT), peso de 1000 sementes (PMS), e número de sementes por legume (NSL) em função dos tratamentos avaliados (Tabela 2).

Tabela 2 - Análise de variância para produtividade (PRT), peso de 1000 sementes (PMS) e número de sementes por legume (NSL) sob o efeito de doses de Si.

\begin{tabular}{lclll}
\hline & \multicolumn{3}{c}{ Quadrados médios } \\
\hline FV & GL & PRT & PMS & NSL \\
\hline Blocos & 2 & 112175,4848 & 26,3030 & 0,0017 \\
Tratamentos & 10 & $127847,6545^{\mathrm{ns}}$ & $22,8242^{\mathrm{ns}}$ & $0,0245^{\mathrm{ns}}$ \\
Erro & 20 & 56353,5181 & 16,3696 & 0,0107 \\
\hline CV $(\%)$ & & 7,43 & 2,67 & 4,56 \\
\hline Média & 3197 & 151 & 2,27 \\
\hline
\end{tabular}

ns Não significativo

Verifica-se que a produtividade apresentou uma variação de 2.923 a $3.617 \mathrm{~kg} \mathrm{ha}^{-1}$ (Tabela 3). Em relação ao peso de 1000 sementes e o número de sementes por legume as variações observadas encontram-se no intervalo de 146 a $155 \mathrm{~g}$ e 2,07 a 2,51, respectivamente, estando dentro do esperado para a cultura da soja (Tabela 3 ). 
Tabela 3 - Médias de produtividade (PRT), peso de 1000 sementes (PMS), número de sementes por legume (NSL) sob o efeito de doses crescentes de $\mathrm{Si}$.

\begin{tabular}{cccc}
\hline Tratamentos & $\begin{array}{c}\text { PRT } \\
k^{2} \mathrm{ha}^{-1}\end{array}$ & $\begin{array}{c}\text { PMS } \\
\mathrm{g}\end{array}$ & NSL \\
\hline 0 & $2931 \mathrm{a}$ & $153 \mathrm{a}$ & $2,38 \mathrm{a}$ \\
50 & $2923 \mathrm{a}$ & $151 \mathrm{a}$ & $2,37 \mathrm{a}$ \\
100 & $3311 \mathrm{a}$ & $150 \mathrm{a}$ & $2,51 \mathrm{a}$ \\
150 & $3089 \mathrm{a}$ & $153 \mathrm{a}$ & $2,45 \mathrm{a}$ \\
200 & $3173 \mathrm{a}$ & $153 \mathrm{a}$ & $2,19 \mathrm{a}$ \\
250 & $3246 \mathrm{a}$ & $155 \mathrm{a}$ & $2,22 \mathrm{a}$ \\
300 & $3201 \mathrm{a}$ & $147 \mathrm{a}$ & $2,28 \mathrm{a}$ \\
350 & $3292 \mathrm{a}$ & $146 \mathrm{a}$ & $2,33 \mathrm{a}$ \\
400 & $3008 \mathrm{a}$ & $155 \mathrm{a}$ & $2,15 \mathrm{a}$ \\
450 & $3376 \mathrm{a}$ & $152 \mathrm{a}$ & $2,07 \mathrm{a}$ \\
500 & $3617 \mathrm{a}$ & $152 \mathrm{a}$ & $2,16 \mathrm{a}$ \\
\hline
\end{tabular}

Médias seguidas pela mesma letra não diferem estatisticamente entre si pelo teste de Tukey a 5\%.

Considerando a média nacional da safra 2005/06 que foi de $2.419 \mathrm{~kg} \mathrm{ha}^{-1}$, constata-se que todos os tratamentos inclusive a testemunha (dose 0) superaram esse valor médio. No caso da testemunha, o aumento da produtividade foi de $21,16 \%\left(512 \mathrm{~kg} \mathrm{ha}^{-1}\right)$ em relação à média brasileira. Quando se compara a produtividade média dos tratamentos adubados com Si (3.224 kg ha-1), com a média nacional $\left(2.419 \mathrm{~kg} \mathrm{ha}^{-1}\right)$ verifica-se um aumento de $33,27 \%\left(805 \mathrm{~kg} \mathrm{ha}^{-1}\right)$, comprovando que a região é propícia para o cultivo da soja. Trabalhos realizados por Rezende \& Carvalho (2007), utilizando cultivares de soja nessa mesma região, obtiveram resultados até mais expressivos. Com a produtividade variando de 2.081 a $4.395 \mathrm{~kg} \mathrm{ha}^{-1}$, com destaque para as cultivares BRS MG 68, Paiaguás e AV 2056-7, que proporcionaram rendimentos de 4.395, 3.898 e $3.780 \mathrm{~kg} \mathrm{ha}^{-1}$, respectivamente.

Embora as aplicações de doses crescentes de $\mathrm{Si}$ não tenham apresentado aumentos significativos para a produtividade, verifica-se que a média dos tratamentos adubados $\left(3.224 \mathrm{~kg} \mathrm{ha}^{-1}\right)$ superaram a testemunha em $10 \%$ (2.931 kg ha-1). No caso da soja, a influência do Si no seu desenvolvimento é pouco conhecida até o presente momento, fato que não ocorre com as gramíneas. De acordo com Raij \& Camargo (1973), resultados positivos com a aplicação desse elemento são comumente observados em plantas acumuladoras de $\mathrm{Si}$, como ocorre com a maioria das gramíneas (arroz, cana-de-açúcar, sorgo, milheto, milho, entre outros). Por sua vez, Marschner (1995) concluiu que a absorção e distribuição radial de $\mathrm{Si}$, por meio das raízes da soja para os vasos do xilema são mais restritas a altas concentrações de $\mathrm{Si}$, indicando um mecanismo efetivo de exclusão.

Pesquisadores têm comprovado a ausência de resposta do Si em certas culturas. Pereira et al. (2003) observaram um maior teor de Si em plantas de tomateiro; no entanto, esse aumento não foi capaz de ser traduzido em aumento de produtividade. Lana et al. (2003) apesar de observarem uma relação direta entre o aumento nas doses de silicato de cálcio e o acúmulo de Si no solo ou foliar, não verificaram respostas das doses crescentes de silicato de cálcio na produtividade da cultura do tomateiro. Franzot et al. (2005), com o objetivo de avaliar, em casa de vegetação, o crescimento e a produção de grãos de feijoeiro comum, submetido a doses de Si aplicadas via foliar, observaram que, para as doses empregadas, que variaram de 0 a 17,00 ppm de $\mathrm{Si}$, não ocorreu efeito significativo para a produtividade.

No caso específico da soja, sendo considerada uma planta intermediária no acúmulo de $\mathrm{Si}$, as respostas à aplicação desse elemento são também mais difíceis de serem observadas. Nessa situação, as alterações de metodologia de colocação desse elemento à disposição da planta, como aumento de dose, forma de aplicação (sulco, cobertura e adubação foliar), poderiam constituirse em uma nova alternativa de fornecimento desse elemento às plantas, no sentido de se obter resultados positivos com essa aplicação.

As características (altura, inserção e número de legume por planta) foram alteradas significativamente em função dos tratamentos utilizados (Tabela 4).

Tabela 4 - Análise de variância para número de legume por planta (NLP), altura das plantas (AP), e da inserção do primeiro legume (AIL) sob o efeito de doses crescentes de Si.

\begin{tabular}{|c|c|c|c|c|}
\hline \multicolumn{5}{|c|}{ QUADRADOS MÉDIOS } \\
\hline FV & GL & NLP & AP & AIL \\
\hline Blocos & 2 & 229,93 & 6,63 & 83,1212 \\
\hline Tratamentos & 10 & $359,66 * *$ & $81,75 * *$ & $31,6545 * *$ \\
\hline Erro & 20 & 73,53 & 11,50 & 8,6545 \\
\hline $\mathrm{CV}(\%)$ & & 16,28 & 3,94 & 14,43 \\
\hline Média & & 53 & 86 & 20 \\
\hline
\end{tabular}

** significativo a $1 \%$ de probabilidade pelo teste $\mathrm{F}$.

Verifica-se que ocorreu uma variação de 39 a 75, 80 a $96 \mathrm{~cm}$ e de 15 a $24 \mathrm{~cm}$ para as características número de legume por planta, altura de planta e altura de inserção do 
primeiro legume, respectivamente (Tabela 5). O número de legumes por planta, importante componente da produtividade da soja, foi aumentado significativamente com a aplicação de $200 \mathrm{~kg} \mathrm{ha}^{-1}$ de silicato. Esses resultados são considerados satisfatórios, levando-se em conta as produtividades alcançadas, embora essas características não tenham contribuído muito para se obter resultados significativos na produtividade.

Tabela 5 - Médias de número de legumes por planta (NLP), altura das plantas (AP), altura de inserção do primeiro legume (AIL) sob efeito de doses crescentes de Si, UFLA, 2007.

\begin{tabular}{cccc}
\hline $\begin{array}{c}\text { Tratamentos } \\
\left(\mathrm{kg} \mathrm{Si} \mathrm{ha}^{-1}\right)\end{array}$ & N Legume & $\begin{array}{c}\text { Altura } \\
(\mathrm{cm})\end{array}$ & $\begin{array}{c}\text { Inserção } \\
(\mathrm{cm})\end{array}$ \\
\hline 0 & $62 \mathrm{ab}$ & $80 \mathrm{c}$ & $15 \mathrm{c}$ \\
50 & $46 \mathrm{~b}$ & $82 \mathrm{c}$ & $16 \mathrm{bc}$ \\
100 & $52 \mathrm{ab}$ & $83 \mathrm{c}$ & $17 \mathrm{abc}$ \\
150 & $57 \mathrm{ab}$ & $83 \mathrm{c}$ & $19 \mathrm{abc}$ \\
200 & $75 \mathrm{a}$ & $83 \mathrm{c}$ & $21 \mathrm{abc}$ \\
250 & $59 \mathrm{ab}$ & $83 \mathrm{c}$ & $23 \mathrm{abc}$ \\
300 & $41 \mathrm{~b}$ & $84 \mathrm{bc}$ & $24 \mathrm{a}$ \\
350 & $41 \mathrm{~b}$ & $88 \mathrm{abc}$ & $24 \mathrm{ab}$ \\
400 & $56 \mathrm{ab}$ & $91 \mathrm{abc}$ & $21 \mathrm{abc}$ \\
450 & $39 \mathrm{~b}$ & $96 \mathrm{a}$ & $22 \mathrm{abc}$ \\
500 & $51 \mathrm{ab}$ & $94 \mathrm{ab}$ & $22 \mathrm{abc}$ \\
\hline
\end{tabular}

Médias seguidas pela mesma letra não diferem estatisticamente entre si pelo teste de Tukey a 5\%.

Em relação à altura da planta e da inserção do $1^{\circ}$ legume, verifica-se que essas características apresentaramse dentro dos padrões normais para a colheita mecânica. A inserção do $1^{\circ}$ legume apresentou menor altura, com média de $15 \mathrm{~cm}$, que ainda assim permite a colheita de forma eficiente.

Pode-se ressaltar que a variação na altura das plantas de 80 a $96 \mathrm{~cm}$, que corresponde, respectivamente, à testemunha e à dose de $450 \mathrm{~kg} \mathrm{ha}^{-1}$ de $\mathrm{Si}$ obtidas no ensaio, indica que não ocorreu nenhum efeito fitotóxico em função dos tratamentos utilizados e, sim, um aumento de $20 \%(16 \mathrm{~cm})$

\section{CONCLUSÕES}

As doses de Si utilizadas não proporcionaram aumentos significativos na produtividade de grãos, peso de mil sementes, número de sementes por legume.

Com o aumento das doses verifica-se aumento significativo no número de legumes por planta, altura de plantas e altura de inserção do primeiro legume.

\section{AGRADECIMENTO}

À FAPEMIG (Fundação de Amparo à Pesquisa do Estado de Minas Gerais), que promove atividades de fomento, apoio e incentivo a pesquisas científicas e tecnológicas em Minas Gerais, possibilitando a realização do estudo por meio do fornecimento de auxílio financeiro.

\section{REFERÊNCIAS BIBLIOGRÁFICAS}

BRASIL. Ministério da Agricultura e Reforma Agrária. Regras para análise de sementes. Brasília: SNDA/ DNPV/CLAV, 1992. 365p.

COMPANHIA NACIONAL DEABASTECIMENTO. Indicadores da agropecuária: ano XVI. Brasília, 2007.

DATNOFF, L.E.; SNYDER, G.H.; KORNDORFER, G.H. Silicon in agriculture. New York: Academy, 201. 403p.

EMPRESA BRASILEIRA DE PESQUISA

AGROPECUÁRIA. Centro Nacional de Pesquisa de Solos. Manual de métodos de análises de solo. 2.ed. Rio de janeiro, 1997. 212p. (Documento, 1).

FERREIRA, R.S. Influência do Si na indução de resistência à mosca-branca Bemisia tabaci biótipo $\mathrm{B}$ (Genn.) (Hemíptera: Aleyrodidae) e no desenvolvimento vegetativo em dois cultivares de soja Glycine max (L.) Merrill. 2006. 40p. Dissertação (Mestrado)Universidade Federal de Lavras, Lavras, 2006.

FRANZOT, B.P.; SILVEIRA, L.S.M. da; ANDRADE, M.J.B. de; VIEIRA, N.M.B.; SILVA, V.M.P. e; CARVALHO, J.G. de. Aplicação foliar de Si em feijoeiro comum. In: CONGRESSO NACIONAL DE PESQUISA DE FEIJÃO, 8., 2005, Goiânia. Anais... Goiânia, 2005. p.957960.

JULIATTI, F.C.; RODRIGUES, F.A.; KORNDÖRFER, G.H.; SILVA, O.A.; CORRÊA, G.F.; PEIXOTO, J.R. Efeito da aplicação de Si na indução de resistência a Diaporthe phaseolorum f. sp. meridionalis em cultivares de soja com diferentes níveis de resistência. Fitopatologia Brasileira, Brasília, v.21, p.51, 1996. Suplemento.

KILMER, V.J. Silicon. In: BLACK, C.A. (Ed.). Methods of soil analysis: chemical and microbiological properties. Madison: American Society of Agronomy, 1965. v.2, p.959-962. (Agronomy, 9). 
LANA, R.M.Q.; KORNDÖRFER, G.H.; ZANÃO JÚNIOR, L.A.; SILVA, A.F.; LANA, A.M.Q. Efeito do silicato de cálcio sobre a produtividade e acumulação de Si no tomateiro. Bioscience Journal, Uberlândia, v.19, n.2, p.15-20, 2003.

LIMA, L.M. Manejo da ferrugem da soja (Phakopsora pachyrhizi Sydow \& P. Sydow). 2006. 81p. Dissertação (Mestrado)-Universidade Federal de Lavras, Lavras, 2006.

LIMA, M.T.G. Interrelação cancro da haste (Diaporthe phaseolorum f. sp. meridionalis), nodulação (Bradyrhizobium japonicum) $e$ Si em soja (G1vcine Max (L) Merril). 1998. 58p. Tese (Doutorado em Energia Nuclear na Agricultura)-Escola Superior de Agricultura "Luiz de Queiroz", Piracícaba, 1998.

MA, J.; TAKAHASHI, E. Effect of silicate on phosphate availability for rice in a P-deficient soil. Plant and Soil, v.133, n.2, p.151-155, 1991.

MARSCHNER, H. Mineral Nutrition of higher plants. 2.ed. New York: Academic, 1995. 887p.

MIYAKE, Y.; TAKAHASHI, E. Effect of silicon on the growth of soybean in a solution culture. Soil Science and Plant Nutrition, Tokyo, v.31, p.625-636, 1985.

NASCIMENTO, J.F.; ZAMBOLIM, L.; DUARTE, H.S.S.; RODRIGUES, F.A. Effect of potassium silicate combine dor not with systemic or protector fungicides on the control fo asian soybean rust. In: DATNOFF, L.E.; SNYDER, G.H.; KORNDÖRFER, G.H. Silicon in agriculture. Amsterdam: Elsevier, 2005. p.121.
NOLLA, A.; KORNDÖRFER, G.H.; ARRUDA, D.G. Acumulação de Si na soja sob diferentes níveis de calcário e silicato. In: SIMPÓSIO CIENTÍFICO DO INSTITUTO DE CIÊNCIAS AGRÁRIAS DA UNIVERSIDADE FEDERAL DE UBERLÂNDIA, 1., 2004, Uberlândia. Anais... Uberlândia: UFU, 2004.

PEREIRA, H.S.; VITTI, G.C.; KORNDÖRFER, G.H. Comportamento de diferentes fontes de Si no solo e na cultura do tomateiro. Revista Brasileira de Ciências do Solo, Viçosa, v.27, n.1, p.101-108, 2003.

RAIJ, B.V.; CAMARGO, O.A. Silica solúvel em solos. Bragantia, Campinas, v.32, n.11, p.223-236, 1973.

REZENDE, P.M. de; BUENO, L.C. de; SEDIVAMA, T.; JUNQUEIRA NETTO, A.; LIMA, L.A. de P.; FRAGA, A.C. Épocas de desbaste em experimentos com soja (Glycine max L. Merrill) em diferentes densidades de semeadura. In: SEMINÁRIO NACIONAL DE PESQUISA DE SOJA, 2., 1981, Brasília. Anais... Londrina: Embrapa/ CNPSoja, 1981. p.14.

REZENDE, P.M. de; CARVALHO, E.A. Avaliação de cultivares de soja [Glycine max (L.) Merril] para o sul de Minas Gerais. Ciência e Agrotecnolgia, Lavras, v.31, n.6, p.1616-1623, nov./dez. 2007.

RIBEIRO, A.C.; GUIMARÃES, P.T.G.; VICENTE, V.H.A. Recomendações para o uso de corretivos e fertilizantes em Minas Gerais: $5^{a}$ aproximação. Lavras: UFLA, 1999. 359p.

SAVANT, N.K.; KORNDORFER, G.H.; DATNOFF, L.E.; SNYDER, G.H. Silicon nutrition and sugarcane production: a review. Journal of Plant Nutrition, v.22, n.12, p.1853-1903, 1999. 